\title{
Backtracking-based Load Balancing
}

\author{
Tasuku Hiraishi \\ Academic Center for Computing and Media Studies, \\ Kyoto University \\ \{tasuku\}@media.kyoto-u.ac.jp
}

\author{
Masahiro Yasugi Seiji Umatani \\ Taiichi Yuasa \\ Graduate School of Informatics, Kyoto University \\ \{yasugi, umatani, yuasa\}@kuis.kyoto-u.ac.jp
}

\begin{abstract}
High-productivity languages for parallel computing become more important as parallel environments including multicores become more common. Cilk is such a language. It provides good load balancing for many applications including irregular ones; that is, it keeps all workers busy by creating plenty of "logical" threads and adopting the oldest-first work stealing strategy. This paper proposes a "logical thread"-free framework called Tascell, which achieves a higher performance and supports a wider range of parallel environments including clusters without loss of productivity. A Tascell worker spawns a "real" task only when requested by another idle worker. The worker performs the spawning by temporarily "backtracking" and restoring its oldest task-spawnable state. Our approach eliminates the cost of spawning/managing logical threads. It also promotes the reuse of workspaces and improves the locality of reference since it does not need to prepare a workspace for each concurrently runnable logical thread. Furthermore, Tascell enables elegant and highly-efficient backtrack search algorithms with delayed workspace copying. For instance, our 16-queens problem solver is 1.86 times faster than Cilk on a system with two dual-core processors. Our approach also enables a single program to run in both shared and distributed memory environments with reasonable efficiency and scalability.
\end{abstract}

Categories and Subject Descriptors D.3.3 [PROGRAMMING LANGUAGES]: Language Constructs and Features-Concurrent programming structures

\section{General Terms Design, Languages, Performance}

Keywords load balancing, parallel computing, backtracking, backtrack search

\section{Introduction}

For efficient parallel computing, all computing resources-such as cores in cluster nodes-in a system are expected to have their own work at any given time. However, it is difficult to predict appropriate work allocation statically in heterogeneous or dynamically varying environments involving multitasking operating systems and/or dynamically joining/leaving computing resources. Irregular applications, such as tree-recursive algorithms and backtrack search algorithms, also make the prediction difficult. In such

Permission to make digital or hard copies of all or part of this work for personal or classroom use is granted without fee provided that copies are not made or distributed for profit or commercial advantage and that copies bear this notice and the full citation on the first page. To copy otherwise, to republish, to post on servers or to redistribute to lists, requires prior specific permission and/or a fee.

PPoPP'09, February 14-18, 2009, Raleigh, North Carolina, USA.

Copyright (C) 2009 ACM 978-1-60558-397-6/09/02 . .\$5.00 cases, dynamic load balancing, where a task (a piece of work) is dynamically allocated to idle computing resources, is effective. Note that the entire computation should be divided into larger tasks in order to reduce the total division/allocation costs.

For shared memory environments, Cilk [5] provides good load balancing in addition to award-winning overall productivity [11]. It succeeds in keeping all workers busy by creating plenty of "logical" threads and adopting the oldest-first work stealing strategy. Creation of logical threads and their synchronization can simply be specified with the keywords spawn and sync as extensions to C. Workers are $O S$ threads provided as virtual computing resources. Usually, the number of workers does not exceed the number of underlying computing resources so that workers actually run in parallel. Cilk employs the implementation technique called LTC (Lazy Task Creation) [12], in which each worker spawns plenty of logical threads and schedules them internally and thus efficiently. An idle worker (thief) may steal a logical thread from another worker (victim). That is, logical threads are used as tasks dynamically allocated to idle workers. When a logical thread recursively spawns offspring logical threads, the adoption of the oldest-first work stealing strategy is effective in making tasks larger.

This paper proposes a "logical thread"-free framework called Tascell, which achieves a higher performance and supports a wider range of parallel environments including clusters without loss of productivity. A Tascell worker spawns a "real" task only when requested by another idle worker. The worker performs the spawning by temporarily "backtracking" and restoring its oldest taskspawnable state.

The contributions of this paper are twofold:

- We propose a new idea for dynamic load balancing, which is based on "backtracking" and does not use "logical" threads.

A worker performs a computation sequentially with an ability to perform backtracking-based temporary restoration of taskspawnable states.

- We discuss how to implement this new idea as an efficient dynamic load balancing programing/execution framework. We also discuss its effectiveness in terms of suitable applications and environments.

Our approach eliminates the cost of spawning/managing logical threads. It also promotes the reuse of workspaces and improves the locality of reference since it does not need prepare a workspace for each concurrently runnable logical thread.

Our framework allows programmers to write undo-redo operations to be executed in backtracking. This enables elegant and highly-efficient backtrack search algorithms with delayed workspace copying. For instance, our 16-queens problem solver is 1.86 times faster than Cilk on a system with two dual-core processors. 


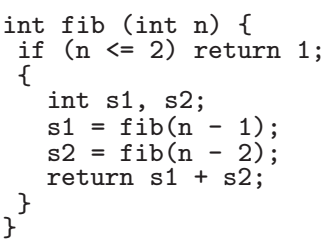

Figure 1. C program for Fibonacci.

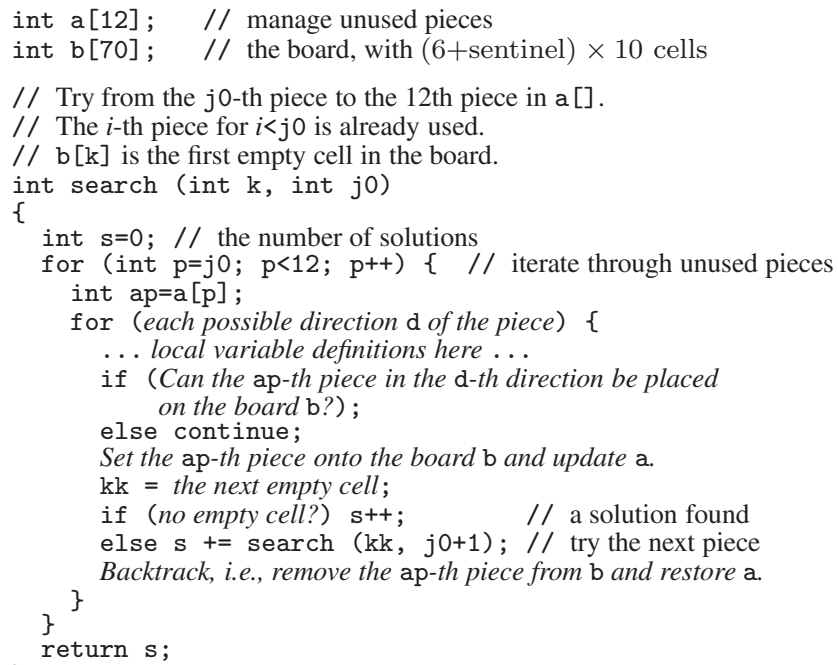

Figure 2. C program for Pentomino.

In addition, we adopted message passing for communication among workers that exchange tasks and results as serialized task objects. This allows a single program to run in both shared and distributed (and also hybrid) memory environments with reasonable efficiency and scalability.

\section{Motivation}

We introduce two tree-recursive algorithms as examples. These algorithms are traditionally difficult to parallelize with low overhead, and our approach is effective (even) for them. Note that the second example supports parallel loops in a tree-recursive manner. By using these examples, we discuss the difficulty in this section and explain the details of our proposal in the next section.

The first example is a doubly recursive algorithm for computing the $n$-th term of the Fibonacci sequence. Figure 1 shows a sequential $\mathrm{C}$ program for it. In each function call, computations of $\mathrm{fib}(n-1)$ and $\mathrm{fib}(n-2)$ can be executed in parallel. This algorithm has no practical meaning, but this example is often used (e.g., in $[3,4,5,6,9,12,18,19])$ for evaluating parallel languages; since each function call has little actual work, the measured overhead well represents the worst case overhead for similar tree-recursive algorithms.

The second example is a search algorithm for finding all possible solutions to the Pentomino puzzle. A pentomino consists of five squares attached edge-to-edge. There are twelve pentominos of different shapes. The Pentomino puzzle involves filling the $6 \times 10$ rectangular board with the twelve pentominos. This problem represents many similar search problems. Figure 2 shows a sequential C program for this problem. Each function call iterates through unused pieces (the outermost loop) and their directions (the inner loop). Parallelization seems applicable to the outermost loop. How-

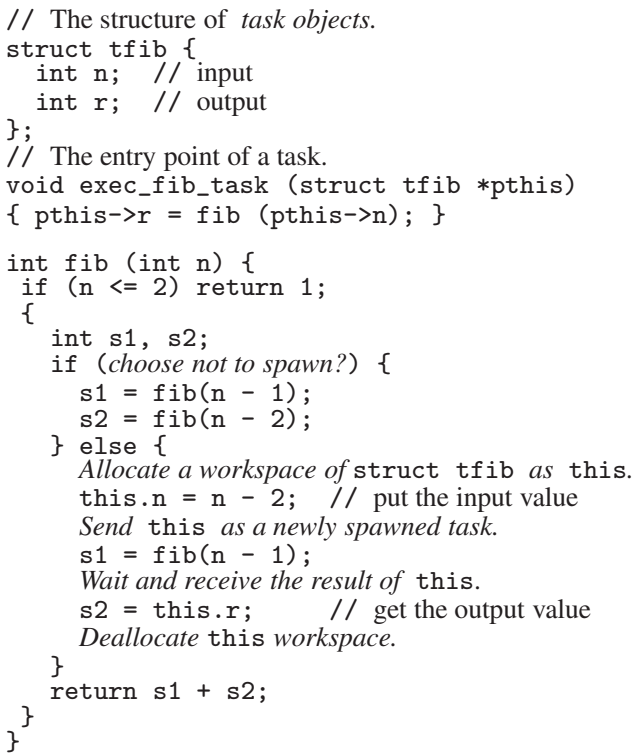

Figure 3. Straightforward task-parallel program for Fibonacci.

ever, there is an important difference from the Fibonacci program; this program does backtrack search where states of the board and the pieces are stored in workspaces: a piece is set at the next available position by one-step extension and removed by backtracking.

One might simply think that one can achieve efficient load balancing by restricting task creation according to the "latest" state (e.g., the number of spawned tasks). Such a straightforward taskparallel program for Fibonacci can be written as in Figure 3. In $\mathrm{fib}(n)$, each worker chooses whether it executes $\mathrm{fib}(\mathrm{n}-2)$ by itself or it spawns a fib(n-2) task. For efficient load balancing, each task should be as large as possible so that a minimum sufficient number of tasks are created to keep all workers busy during the entire running time. That is, for each task, its worker should choose to spawn a proper number of tasks in the early stage and then choose not to spawn any more tasks except for adjusting the completion time. Such a strategy is infeasible without precise information (prediction) about the entire execution (not the "latest" information at the choice point). Thus, this straightforward approach does not work.

Figure 4 shows a straightforward task-parallel program for Pentomino. For parallelization, the outer for loop in Figure 2 is replaced with a PAR_LOOP macro. In PAR_LOOP, each worker chooses whether it performs all iterations or it spawns a task for the upper half of iterations. (In the latter case, it has another choice on the remaining lower half of iterations with a recursive application of the PAR_LOOP macro.) This straightforward approach does not work since it requires an infeasible strategy as in the case of the Fibonacci program.

In the following sections, we propose our approach with a feasible strategy for efficient load balancing. Note that the worker that executes a spawned task often requires its own initialized (copied) workspace as in Figure 4. This motivated us to make an additional innovation in our approach.

\section{Our approach}

We propose a programming and execution framework called "Tascell." Tascell stands for task cell, which indicates that running tasks are divided like biological cells. In Tascell, we can spawn a task lazily by using backtracking. 
// The structure of task objects.

// Each worker has to have its own board for parallelization.

struct pentomino \{

int $\mathrm{s}$; // output

int $\mathrm{k}$, i0, i1, i2;

int $\mathrm{a}[12] ; / /$ manage unused pieces \};

int $\mathrm{b}[70] ; / /$ the board, with $(6+$ sentinel $) \times 10$ cells

exec_pentomino_task (struct pentomino *pthis)

$\{$ pthis->s $=$ search(pthis->k, pthis->i0, pthis->i1, pthis->i2, pthis); \}

\#define PAR_LOOP (_i1, _i2,_body) \{

if (choose not to spawn?) \{

for $\left(; \_i 1<\_i 2 ;\right.$ _i1++) _body

\} else \{

int $i h=(i 1+i 2) / 2$;

int $\bar{i} 1=i \bar{h} ; / /$ range for the new sub-task i1--i2

int i2 = $-i 2$;

Allocate a workspace of struct pentomino as this.

$\{$ // put task inputs for upper half iterations

copy_piece_info (this.a, tsk->a); // copy the

copy_board (this.b, tsk->b); // workspace

this.k $=\mathrm{k}$; this. $\mathrm{iO}=\mathrm{j} 0$

this.i1 = i1; this.i2 = i2;

Send this as a newly spawned task.

// lower half iterations (expanded $n$ times for $n$-bit int)

PAR_LOOP ( i1, ih, body)

Wait and receive the result of this.

$\mathrm{s}+=$ this.s; // get the result

(

Deallocate this workspace.

// Try from the $j 1$-th piece to the $j 2$-th piece in $a[]$.

// The $i$-th piece for $i<j 0$ is already used.

$/ / \mathrm{b}[\mathrm{k}]$ is the first empty cell in the board.

int search (int $k$, int $j 0$, int $j 1$, int $j 2$,

\{ struct pentomino *tsk)

int $s=0 ; / /$ the number of solutions

int $\mathrm{p}=\mathrm{j} 1$

PAR_LOOP $(p, j 2,\{$

int $\mathrm{ap}=\mathrm{tsk}->\mathrm{a}[\mathrm{p}]$;

for (each possible direction $\mathrm{d}$ of the piece) \{

... local variable definitions here ...

if (Can the ap-th piece in the $\mathrm{d}$-th direction be placed on the board $\mathrm{tsk}->\mathrm{b}$ ?)

else continue;

Set the ap-th piece onto the board $\mathrm{tsk}->\mathrm{b}$ and update $\mathrm{ts \textrm {k } - > \mathrm { a }}$ $\mathrm{kk}=$ the next empty cell;

if (no empty cell?) s++; // a solution found

else

$\mathrm{s}+=\operatorname{search}(\mathrm{kk}, j 0+1, j 0+1,12, \mathrm{tsk})$;

Backtrack, i.e., remove the ap-th piece from $\mathrm{tsk}->\mathrm{b}$ \})

and restore $\mathrm{tsk}->\mathrm{a}$.

return s;

\}

Figure 4. Straightforward task-parallel program for Pentomino.

The sequential computation of the $\mathrm{C}$ program in Figure 1 (Figure 2) is outlined as a depth-first, left-to-right traversal of the invocation tree. Notice that the straightforward task-parallel program in Figure 3 (Figure 4) involves the same traversal if its worker always chooses not to spawn a task.

In Tascell, the worker always chooses not to spawn at first, but when it receives a task request, it spawns a task as if it changed the past choice. That is, as is shown in Figure 5 (Figure 6),

1. it backtracks (goes back to the past),

2. it spawns a task (and changes the execution path to receive the result of the task),

3. it returns from the backtracking (restores the time), and

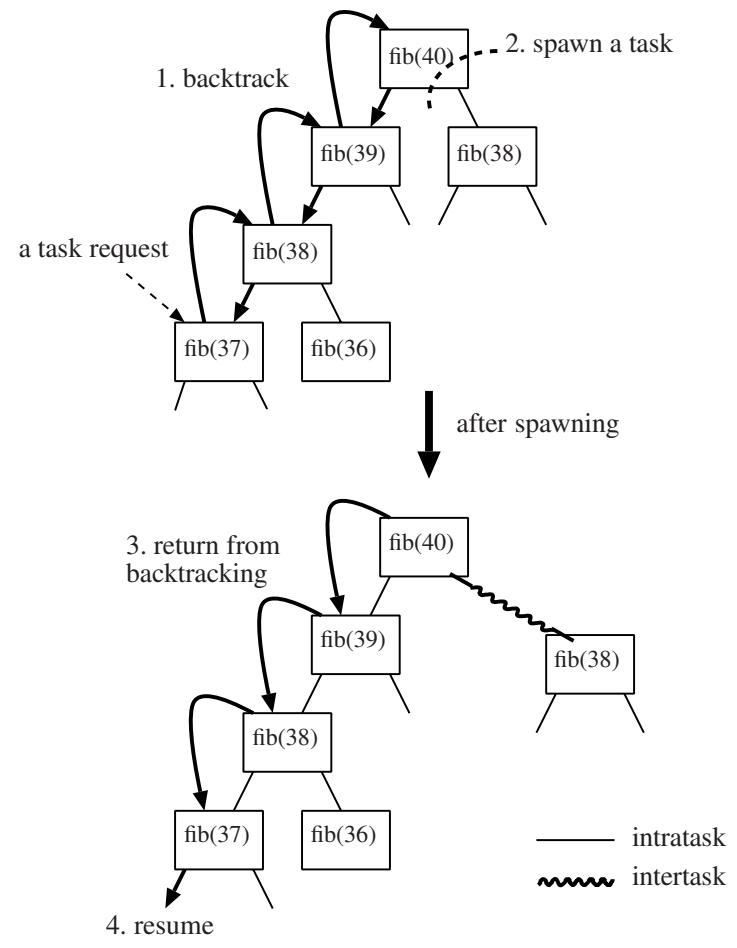

Figure 5. Spawning a task lazily while computing fib(40). When a Tascell worker detects a task request (at fib(37)), it (1) backtracks to the oldest task-spawnable point, (2) spawns a task for fib(38), (3) returns from backtracking, and (4) resumes its own computation.

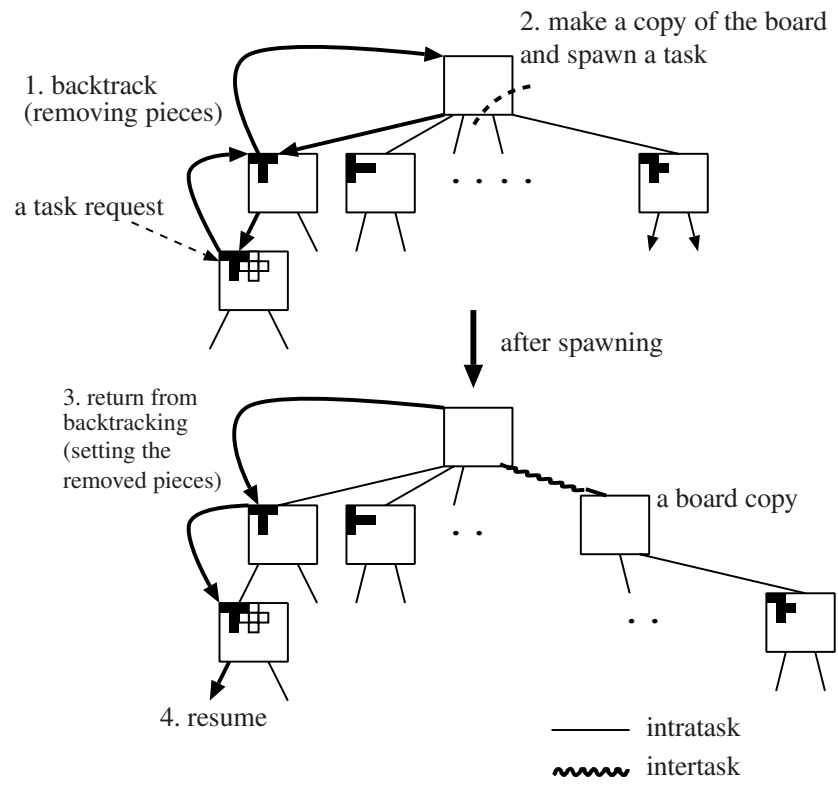

Figure 6. Spawning a task lazily while performing backtrack search for Pentomino. Unlike in Figure 5, (1) the backtracking step includes undo operations (i.e., removing pieces). (2) The spawninghalf-iterations step includes making a copy of the temporarily restored board. (3) The returning-from-backtracking step includes redo operations (i.e., setting pieces). 
4. then it resumes its own task.

Notice that we can spawn a larger task (as is a fib(38) subtree in the lower right part of Figure 5), in general, by backtracking to the oldest task-spawnable choice point. ${ }^{1}$

A Pentomino worker performs a sequential computation efficiently with its own workspace by setting a piece and by removing the piece (i.e., backtracking or undoing) across search steps. When the worker spawns a task, it must copy (part of) the "current" contents of its workspace into a newly allocated space for the new task as in Figure 4. In our approach, the "current" contents should be equal to the past contents at the time of the past choice. As is shown in Figure 6, the worker can recover the past contents by performing proper undo operations along with backtracking as part of Step 1, it spawns a task with a copy of its workspace at Step 2, and then it performs proper redo operations as part of Step 3 in order to resume its own task at Step 4.

To address the problem of load-based inlining, which is essentially the straightforward approach in Section 2, fine-grain multithreaded languages such as Cilk [5] and MultiLisp [7] also use a technique called Lazy Task Creation (LTC) [12]. Our approach differs from LTC in the following manner:

- Our worker performs a sequential computation unless it receives a task request. Because no logical threads are created as potential tasks, the cost of managing a queue for them can be eliminated.

- In multithreaded languages, each (logical) thread requires its own workspace. In contrast, our worker can reuse a single workspace while it performs a sequential computation to improve the locality of reference and achieve a higher performance.

- When we implement a backtrack search algorithm in multithreaded languages, each thread often needs each its own copy of its parent thread's workspace. In contrast, our worker can delay copying between workspaces by using backtracking.

- Our approach supports (heterogeneous) distributed memory environments (including mixed-endian environments) without using distributed shared memory systems.

Note that LTC assumes that the number of really created tasks (and steals) is incomparably smaller than the number of logical threads. Our approach also assumes that the number of really spawned tasks (and steals) is very small. This assumption justifies our approach, which accepts higher work-stealing (backtracking) overheads in order to achieve lower serial overheads than more conventional LTC such as Cilk.

We may use additional constructs in order to specify how to perform backtracking and undo-redo operations. These constructs are detailed in Section 4.2.

\section{Tascell framework}

We designed and implemented a framework to realize our idea. The Tascell framework consists of a Tascell server and a compiler for the Tascell language.

\subsection{Overview}

Figure 7 shows a multistage overview of the Tascell framework. Compiled Tascell programs are executed on one or more computation nodes. Each computation node has one or more worker(s) in

\footnotetext{
${ }^{1}$ Tascell can be extended such that, beyond the statistical assumption, we may spawn larger tasks by individually considering all task-spawnable points using additional expected task information from users, analyzers, and/or profilers.
}

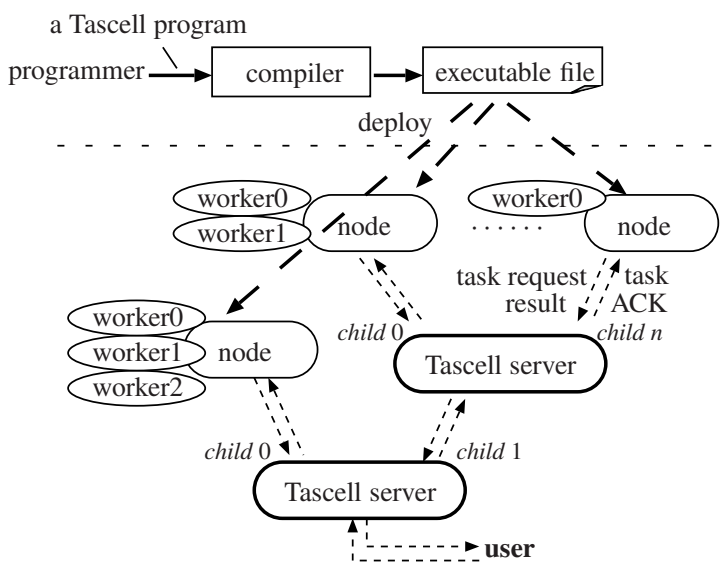

Figure 7. Multistage overview of the Tascell framework.

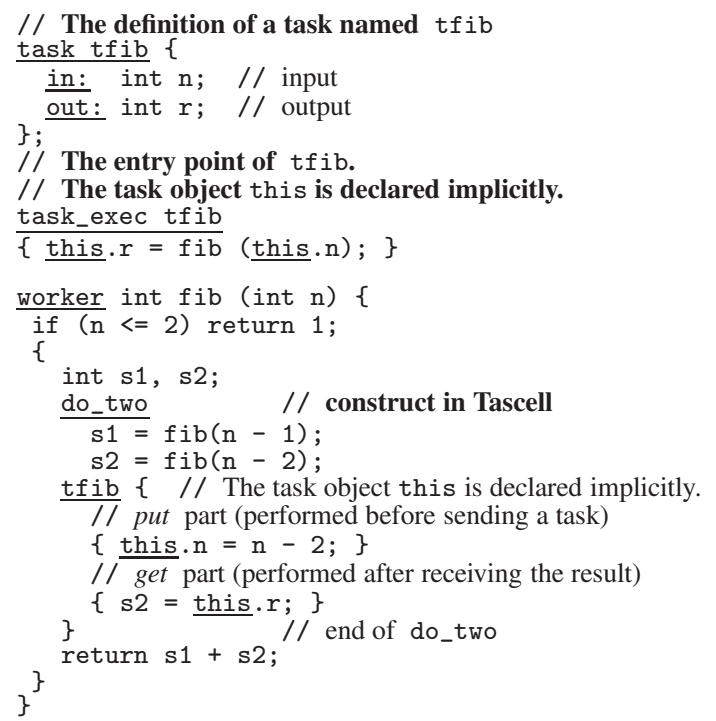

Figure 8. Tascell program for Fibonacci.

the shared memory environment (the number can be specified as a runtime option).

For good load balancing, idle workers should request tasks of loaded workers. An idle worker sends a task request to either a specific worker or any worker. Intranode (Internode) messages are relayed by Tascell runtime systems (Tascell servers), which choose loaded workers (nodes) for "to any" task requests. Such a series of messages is exchanged automatically; programmers need not (and cannot) treat each message directly.

Each task or its result is transmitted as a task object whose structure is defined in a Tascell program. If a request is from the same node, (the pointer to) the object can be passed quickly via shared memory, otherwise the object is transmitted as a serialized message via Tascell servers.

\subsection{Tascell Language}

The Tascell language is an extended $\mathrm{C}$ language. Figures 8 and 9 are examples of Tascell programs. (Tascell extensions are underlined.)

Programmers can write a worker program with new constructs in Tascell, starting with an existing sequential program. Tascell 


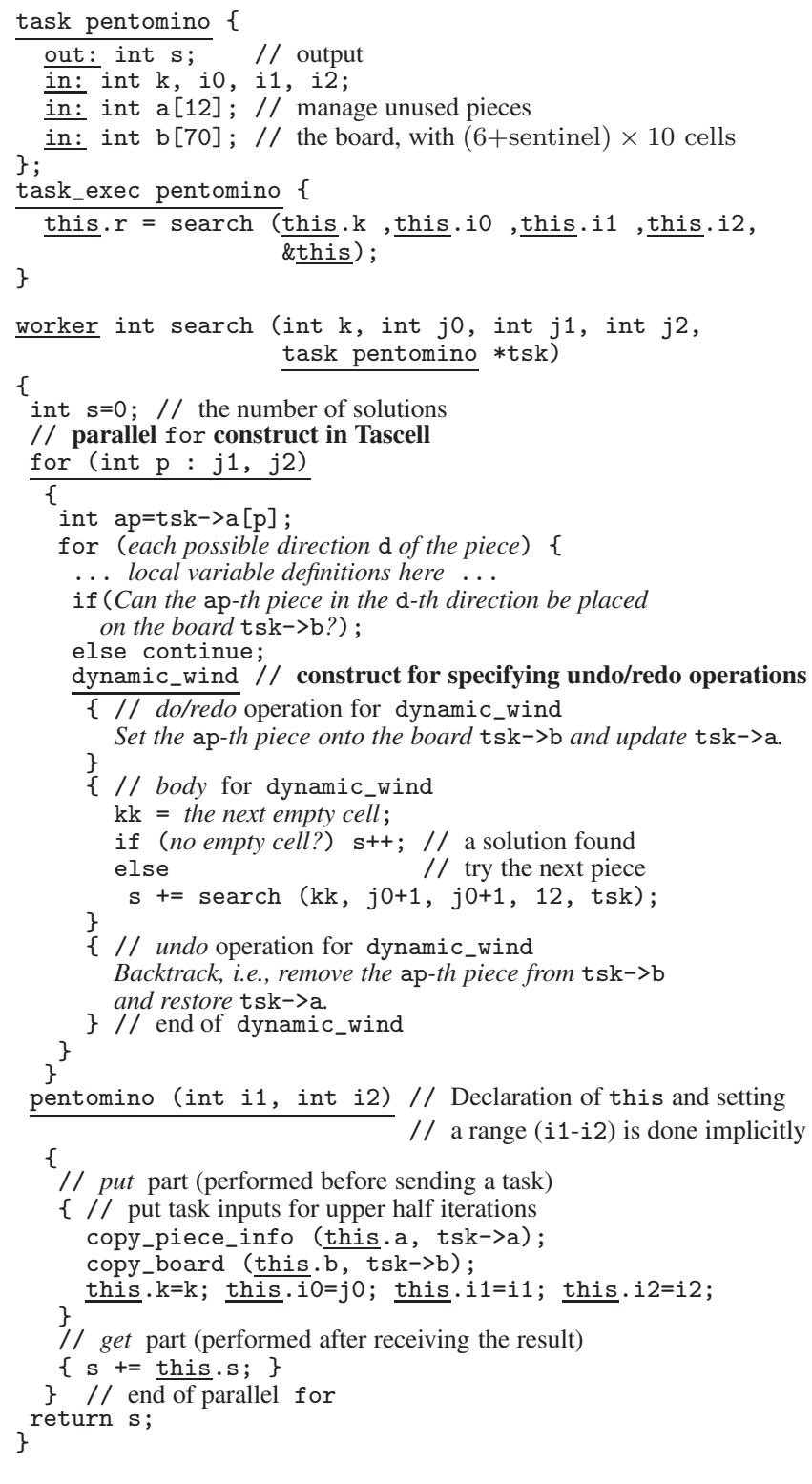

Figure 9. Tascell Program for Pentomino.

has constructs for defining a task and for specifying potential task division with optional temporary undo/redo operations.

\subsubsection{Task definition}

A top-level task declaration:

$$
\text { task task-name }\{[\text { in:|out:] struct-declaration } \cdots\} \text {; }
$$

gives the structure of task-name task objects. For instance, "task tfib \{in: int $n$; out: int $r$ \};" in Figure 8 declares the structure of task tfib objects. The syntax is the same as that for the definitions of structs, except that we may specify an in: or out: attribute for each field. A Tascell compiler uses attributes to construct default send/receive methods of the task. In addition, we can add user-defined send/receive methods in order to skip transmitting (part of) inputs/outputs selectively or allocating/freeing a workspace (the details are omitted in this paper because of space limitation)

\section{Definition of entry points A top level declaration}

$$
\text { task_exec task-name }\{\text { body }\}
$$

defines the computation of a task-name task. In the body ("this.r =fib(this.n);" in Figure 8), we can refer to the task object by the keyword this, which includes an input of the task in some fields, and we should set the result of the computation into appropriate fields. In addition, we can call worker functions in the body.

\subsubsection{Worker functions}

In worker functions, which are specified with the keyword worker (like cilk procedures in Cilk), we can use Tascell's task division constructs as explained below. Only worker functions (and task_exec bodies) can call worker functions.

\subsubsection{Constructs for task division}

A statement:

$$
\begin{aligned}
& \text { do_two } \text { statement }_{1} \text { statement }_{2} \\
& \text { task-name }\left\{\text { statement }_{\text {put }} \text { statement }_{\text {get }}\right\}
\end{aligned}
$$

indicates that a computation in statement 2 (" $\mathrm{fib}(\mathrm{n}-2)$ " in Figure 8) may be spawned during the execution of statement $_{1}$ ("s $1=f i b(n-1)$;" in Figure 8$).^{2}$ More precise steps are as follows:

1 The worker executes statement $t_{1}$ with an implicit task request handler. If it invokes this handler with a task request after backtracking, it divides the current task by spawning a new task-name task, setting the fields of the new task object by statement $_{\text {put }}$ ("this.n=n-2;" in Figure 8), and then sending it to the task requester before returning from the backtracking. Here, a computation in statement 2 is packed as the task object.

2a If the task request handler for do_two is not invoked during the execution of statement $_{1}$, statement 2 is executed.

$2 \mathrm{~b}$ Otherwise, the worker skips statement $_{2}$, waits for the result of the spawned task, and then merges the result by executing statement $_{\text {get }}$ ("s2=this.r;" in Figure 8). In order not to be idle, the worker should request and execute other tasks while waiting for the result. In Tascell, the worker simply requests tasks of the task requester in Step 1 to save its stack size [22].

The identifier task-name specifies the type of a task to be created. The keyword this can be used in statementput and statement $_{\text {get }}$ to refer to the task object. We should initialize a task in statement $_{p u t}$ by assigning values to input fields and can obtain the result of the task in statement get $_{\text {by }}$ referring to output fields. This series of operations should be equivalent to statement 2 .

For dividing an iterative computation, Tascell has a parallel for loop construct syntactically denoted by:

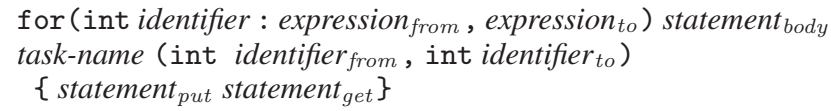

\footnotetext{
${ }^{2}$ Although making work amounts of two statements as equal as possible is a good practice, imbalance and uncertainty are permitted to some degree For example, in a simple situation with two workers, the total amount of unprocessed work decreases rapidly as a product of $\varepsilon_{i}$ when the $i$-th division makes two workers have work amounts in the ratio of $1+\varepsilon_{i}: 1-\varepsilon_{i}$ $\left(0 \leq \varepsilon_{i}<1\right)$
} 
For example, Figure 9 employs a parallel for statement of "for

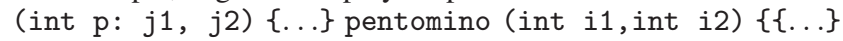
$\{\mathrm{s}+=$ this.s $\}$ \}." This iterates statement body $_{\text {over integers from }}$

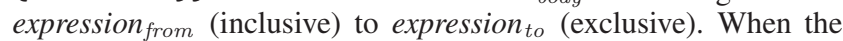
implicit task request handler (available during the iterative execution of statement $t_{b o d y}$ ) is invoked, the upper half of the remaining iterations are spawned as a new task-name task. The actual assigned range can be referred to in statement $_{\text {put }}$ by identifier $_{\text {from }}$

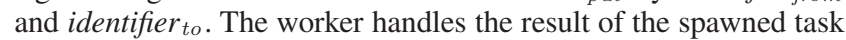
by executing statement get $_{\text {. }}$.

Tascell has a dynamic_wind construct as in the Scheme language [10] for defining undo/redo operations, syntactically denoted by:

dynamic_wind statement before $_{\text {statement }}$ body statement $_{\text {after }}$.

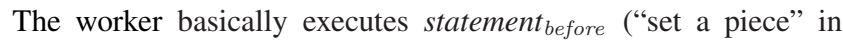

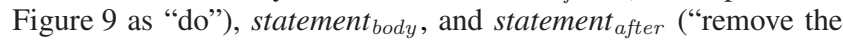
piece" in Figure 9 as "undo") in this order. However, during the execution of statement body $_{\text {, statement }}$ after is also executed as an "undo" clause before an attempt to invoke an older task request handler. Statement before is also executed as a "redo" clause after the attempt.

Backtracking-based task division Do_two, parallel for, and dynamic_wind statements may be nested dynamically in their

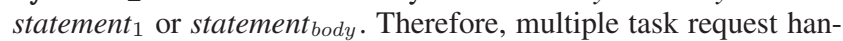
dlers and undo-redo clauses may be available at the same time as in Figures 5 and 6 . Each worker tries to detect a task request by polling at every do_two or parallel for statement. When the worker detects a task request, it performs temporary backtracking in order to spawn a larger task by invoking as old a handler as possible. If there are undo-redo clauses on the backtracking path, undo clauses are executed in turn for the backtracking and redo clauses are executed in turn for the resumption.

\subsubsection{Tascell Programming}

We can write the Tascell program in Figure 8 by (1) starting with the C program in Figure 1, (2) adding the keyword worker to the procedure fib, (3) finding two statements that can be executed in parallel, (4) forming a do_two statement with the consideration of the name and structure of the spawned task, and (5) defining the structure and body of the task.

We can write the Tascell program in Figure 9 by starting with Figure 2 as above, except that (1) we find iterations that can be executed in parallel if separate workspaces are supplied, (2) we form a parallel for statement, (3) we prepare some workspace in the task structure and adjust the access to it, (4) we form a dynamic_wind statement with existing do/undo operations, and (5) we adjust the parameter and body of search in order to accept a task with iterations. Notice that this program avoids undesirable workspace copying and promotes the reuse/sharing of the workspace.

\section{Implementation}

We implemented a Tascell compiler as a translator to the C language in order to make our implementation portable. It is difficult to realize the backtracking mechanism in "standard" $\mathrm{C}$ because it needs "stack walk," accessing variables whose values are located below the current frame in the execution stack. We proposed to implement various language features that require stack walk by using nested functions. We applied this scheme to implement Tascell.

\subsection{Nested functions}

A nested function is a function defined inside another function, in places where variable definitions are allowed except at the top-

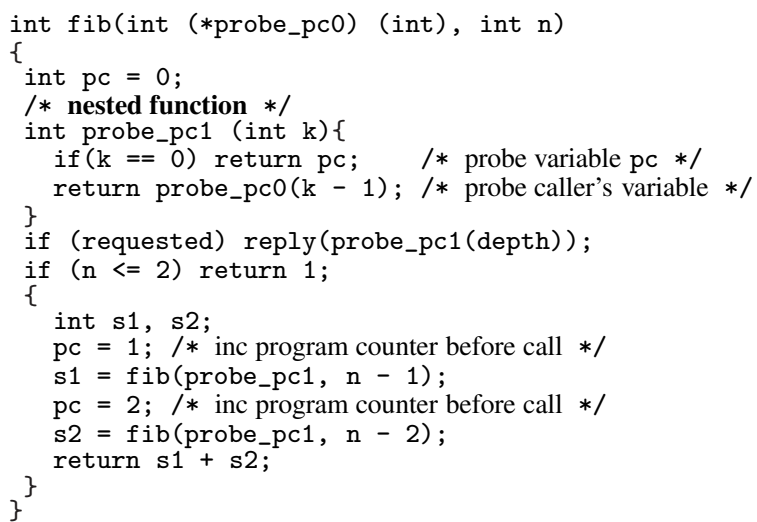

Figure 10. Program with nested functions.

level. Its evaluation creates a lexical closure accompanying the creation-time environment, and indirect calls to it provide legitimate stack access. Figure 10 shows an example of a program with nested functions. When we (indirectly) call the function probe_pc1 nested in $\mathrm{fib}$, we can access a parameter probe_pc0 and a local variable pc locating in the (older) frame. In this example, by using a chain of nested functions, we can probe $p c$ in the depth-th newest frame.

\subsection{Implementations of nested functions}

The most well-known implementation of nested functions for $\mathrm{C}$ is the trampoline-based implementation in GCC $[1,15]$. However, maintenance/creation costs of lexical closures in this implementation are high because it performs runtime code generation and prevents local variables and parameters that are accessed by nested functions from being register-allocated.

Therefore, in our previous work, we realized $L$-closure-based implementations of nested functions. These implementations achieve remarkably low maintenance/creation costs by delaying the initialization of the closure until it is invoked and enabling register allocation. We have two versions of L-closure implementations: a translator to standard C (called $L W-S C$ ) [8] and an enhancement to GCC (called $X C$-cube) [23]. The former allows easy support for various platforms with existing $\mathrm{C}$ compilers, and the latter provides a higher performance by using assembly-level implementation techniques.

\subsection{Translation to $\mathbf{C}$ with nested functions}

The program in Figure 8 is translated to the program in Figure 11 with nested functions. Each worker function is translated to have an additional parameter_bk0 holding a nested function pointer corresponding to the newest handler for a do_two, parallel for, or dynamic_wind statement. Each do_two statement is translated into a piece of code that includes a definition of a nested function (_bk1_do_two in Figure 11) as the newest handler, which is called when a task request is detected by polling. The nested function first tries to spawn a larger task by calling a nested function (_bk0) that corresponds to the second newest handler (which calls another nested function for the third newest handler and so on). Only if a task request still remains, a new task is created and sent to the requester. After sending a task, the worker returns from the nested function and resumes its own computation.

A parallel for statement can be translated in the same way (Figure 12), except that, in the nested function, the worker needs to calculate a range for a new task and update a range for itself. 


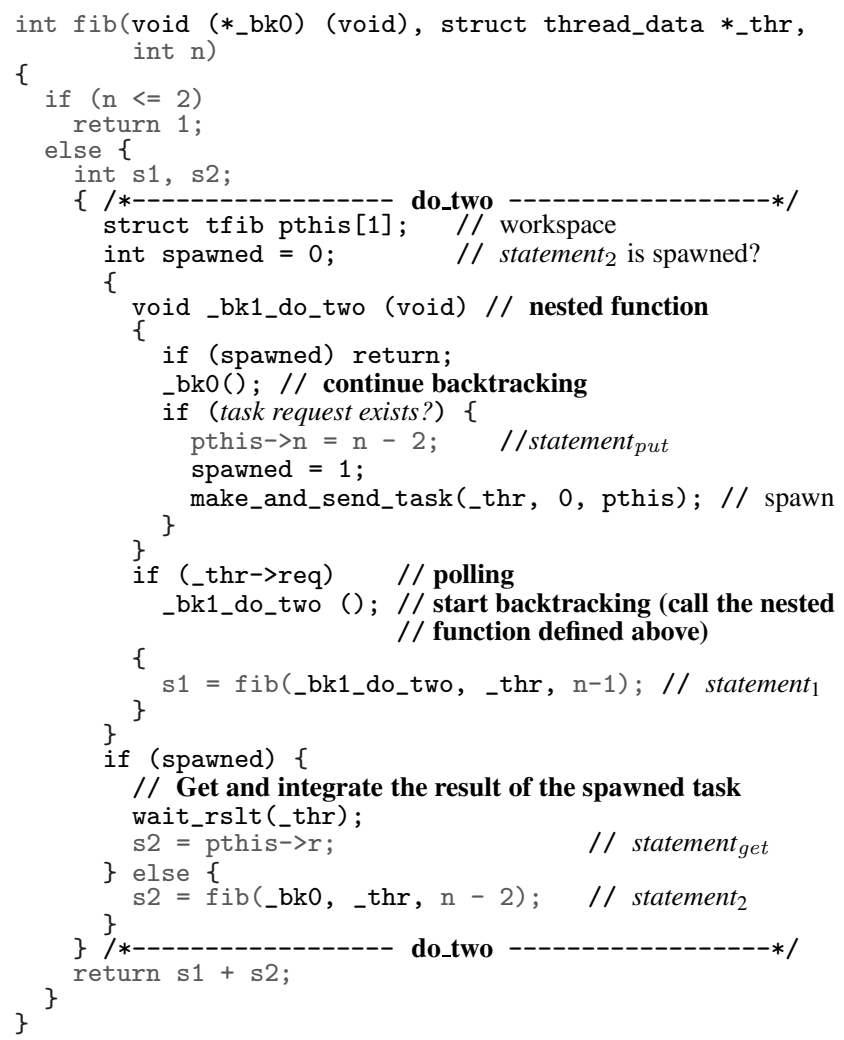

Figure 11. Translation result from the worker function $f i b$ in Figure 8 , including translation of a do_two statement.

Translation for a dynamic_wind statement is also included in

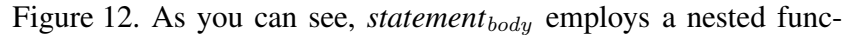
tion (_bk2_dwind in Figure 12), which is composed of (a copy

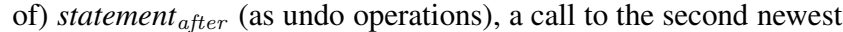
nested function, and (a copy of) statement before (as redo operations), in order to perform undo/redo operations as is described in Section 4.2.3.

\section{Discussion}

This section compares our approach to related work.

\subsection{Multithread-based load balancing}

LTC [12] is one of the best implementation techniques for dynamic load balancing. In LTC, a newly spawned logical thread is directly and immediately executed like a usual call while (the continuation of) the oldest thread in the worker may be stolen by another idle worker. Usually, the idle worker (thief) randomly selects another worker (victim) for stealing a task. Cilk employs this technique.

A message passing implementation [3] of LTC employs a polling method as in Tascell where the victim detects a task request sent by the thief and returns a new task created by splitting the present running task. OPA [19], StackThreads/MP [18], and Lazy Threads [6] employ this technique. Polling methods often improve performance by avoiding "memory barrier" instructions, as Indolent Closure Creation [16] improves Cilk's performance.

WorkCrews [21], Leapfrogging [22], and Lazy RPC [4] take the parent-first strategy; at a fork point, a worker executes the parent thread prior to the child thread and makes the child stealable for other workers, and calls the child thread if it has not been stolen at the join point of the parent thread. Tascell uses a similar strategy;

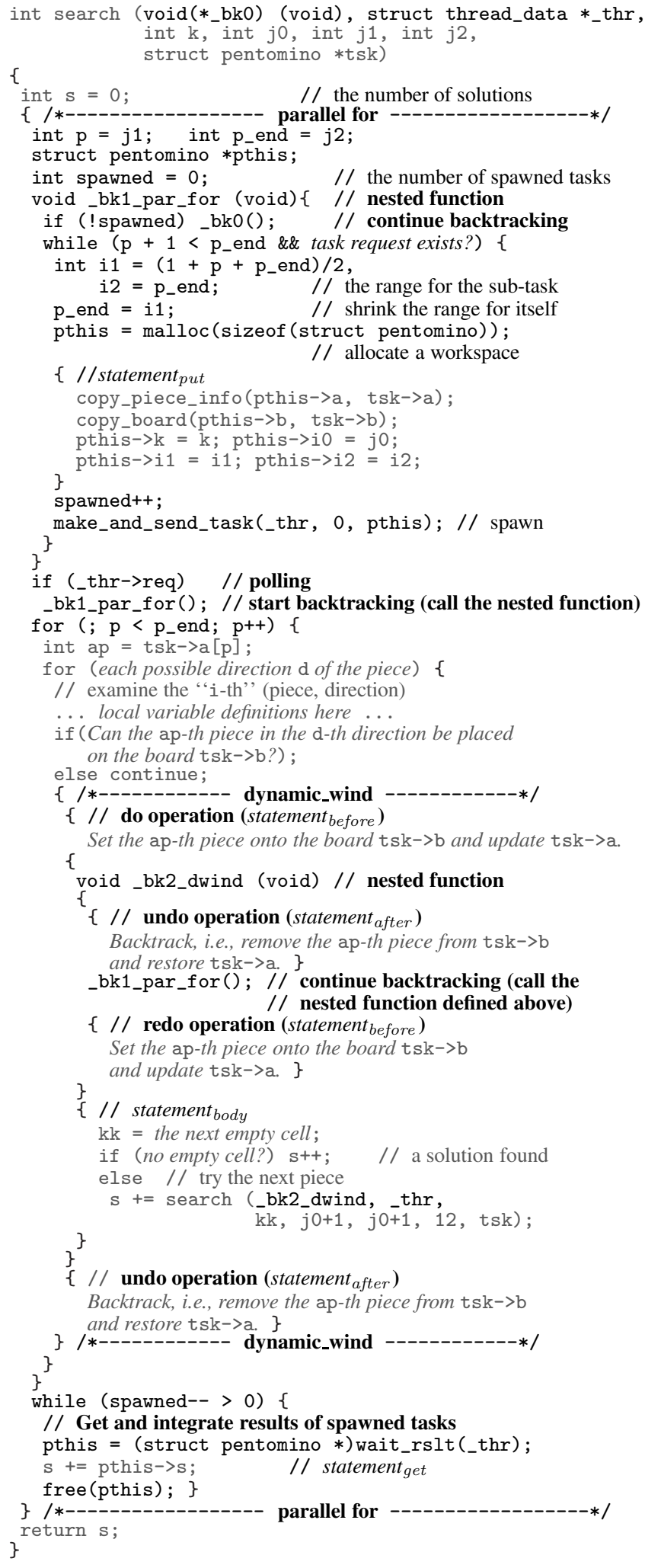

Figure 12. Translation result from the worker function search for Pentomino in Figure 9, including translation of a parallel for statement and a dynamic_wind statement. 
however, creations of stealable entities are delayed and mostly omitted.

Lazy Threads [6] realizes further optimization for spawning a thread by translating it into a parallel ready sequential call. It achieves a lower thread creation cost than the original LTC by avoiding operations for queueing a new thread. However, this technique can be applied only for consecutive forks. Furthermore, it is unclear how this technique can coexist with the oldest-first work stealing strategy.

Our approach is "logical thread"-free, but its ability to restore task-spawnable states without loss of good serial efficiency depends heavily on L-closures and the notion of lazy stack frame management $[8,23]$. The idea of lazy frame management can also be applied to logical threads. Indolent Closure Creation [16] employs this idea for Cilk; its technique of using a shadow stack is similar to the lazy validation of an explicit stack in our transformation-based implementation [8] of L-closures. Moreover, our previous work [19] shows that the notion of "laziness" is effective for modern multithreaded languages with thread IDs and dynamically-scoped synchronizers.

We can find few pieces of recent work that make remarkable advances following the abovementioned techniques; for example, X10's thread (or activity) creation and synchronization are inspired by Cilk, and they do not propose a new technique for load balancing [2]. This means that the LTC/Cilk-originating ideas of "logical threads" for load balancing reach maturity.

Notice that our proposal is to employ different semantics from multithreading rather than to reduce costs for multithreading. Our approach enables further performance improvement by reusing a workspace and delaying copying between workspaces. This is the case in most multithreaded languages other than Cilk. In Cilk, a pseudovariable SYNCHED is provided, which promotes the reuse of a workspace among child logical threads [17]; however, child threads cannot share a workspace with their parent thread.

Except for not using a "logical thread," our usage of multiple workers is quite usual; thus, our framework can be enhanced with existing/new techniques proposed in previous/future work for other aspects of parallel computing, such as duplicate elimination (especially, in search algorithms) and efficient data placement.

\subsection{Distributed memory environment support}

Tascell supports distributed memory environments (including mixedendian environments) by transmitting inputs and outputs as serialized task objects among computation nodes. This support works well if the task size (work amount) is large enough to make the communication cost relatively low. Programmers can write a single program for both shared and distributed memory environments because the interface for passing task objects is integrated. Furthermore, it is easy for new computation nodes to join a running computation dynamically.

Distributed Cilk [14] and SilkRoad [13] employ DSM (Distributed Shared Memory) to support distributed memory environments. DSM is useful to support globally shared data. For this purpose, we may also employ additional libraries or language support.

\subsection{Productivity}

Tascell provides high productivity in the sense that we can write a Tascell program by augmenting an existing $\mathrm{C}$ program, as described in Section 4.2.4. The resulting program would be much simpler than library-based parallelizing frameworks such as TBB [9]. However, when compared to Cilk programs, Tascell programs are more verbose; we need to define tasks and write statements for task inputs/outputs. These costs are necessary for (1) (general) distributed memory environment support and (2) more exact control of workspaces in task objects with and without dynamic_wind.

\begin{tabular}{|l|l|l|}
\hline & Tascell server & $\begin{array}{l}\text { lomputation node } \\
\text { (Tascell/Cilk) }\end{array}$ \\
\hline CPU & $\begin{array}{l}\text { AMD Opteron 244 } \\
1.8 \mathrm{GHz}\end{array}$ & $\begin{array}{l}\text { AMD Dual Core } \\
\text { Opteron 265 1.8GHz } \times 2 \\
\text { (4 cores in total) }\end{array}$ \\
\hline OS & Rocks 4.0 (Linux kernel 2.6.9) \\
\hline compiler & $\begin{array}{l}\text { Allegro Common Lisp 8.1 } \\
\text { with (speed 3) } \\
\text { (safety 1) (space 1) 1) } \\
\text { optimizers }\end{array}$ & $\begin{array}{l}\text { GCC.3 with -02 } \\
\text { optimizers } \\
\text { Tascell compiler + LW-SC } \\
\text { (in Tascell) } \\
\text { Cilk version 5.3 (in Cilk) }\end{array}$ \\
\hline worker & \multicolumn{1}{|c|}{$\begin{array}{l}\text { created by } \\
\text { pthread_create with } \\
\text { PTHREAD_SCOPE_SYSTEM }\end{array}$} \\
\hline $\begin{array}{l}\text { internode } \\
\text { communication } \\
\text { (Tascell) }\end{array}$ & $\begin{array}{l}\text { Each computation node is TCPIP connected to the } \\
\text { single Tascell server on Gigabit Ethernet. } \\
\text { A node having a task does not send a "to any" task } \\
\text { request to another node. }\end{array}$ \\
\hline
\end{tabular}

Table 1. Evaluation environment.

Of course, we may use a more concise description if we limit our language to support only shared memory environments or some limited patterns of parallel computation.

\section{Evaluation}

In this section, we evaluate the performance of the Tascell framework using the following programs:

$\boldsymbol{F i b}(n)$ recursively computes the $n$-th Fibonacci number.

Nqueens $(n)$ finds all solutions to the $n$-queens problem.

$\operatorname{Pentomino}(n)$ finds all solutions to the Pentomino problem with $n$ pieces (using additional pieces and an expanded board for $n>12)$.

$\mathbf{L U}(n)$ computes the LU decomposition of an $n \times n$ matrix.

$\operatorname{Comp}(n)$ compares array elements $a_{i}$ and $b_{j}$ for all $0 \leq i, j<n$.

$\operatorname{Grav}(n)$ computes a total force exerted by $(2 n+1)^{3}$ uniform particles.

Nqueens in Tascell is coded with a combination of a parallel for and a dynamic_wind in the same way as Pentomino. LU and Comp use cache-oblivious recursive algorithms with do_two constructs. A Comp task to compare arrays of size $n$ and $m(n \geq m)$ is divided into two tasks with arrays of $n / 2$ and $m$. Grav is an iterative application. It is implemented in Tascell as triply nested parallel for loops corresponding to three axes. Note that we used finegrained implementations for these applications.

The evaluation environment is summarized in Table 1 .

In order to evaluate serial overheads, we ran the Tascell programs with one worker and compared their execution time with $\mathrm{C}$ and Cilk programs in almost the same algorithms. For Nqueens, Pentomino, and Grav in Cilk, each thread requires its own workspace to hold one or more arrays. Furthermore, for Nqueens and Pentomino, each thread needs its own copy of its parent thread's workspace even when SYNCHED is used, resulting in considerable copying overhead. In Tascell, the worker can reuse a single workspace while it performs a sequential computation as is shown in Section 3.

The results of the performance measurements are shown in Table 2. The overheads in Tascell, which arise from polling and managing nested functions, are considerably lower than Cilk for almost all applications. In particular, Fib shows a sharp contrast in overheads because the frequent creation of logical threads causes a higher overhead in Cilk. Nqueens shows a higher contrast than Pentomino because of more frequent copying. LU shows virtually 


\begin{tabular}{l|r|r|r|r|r} 
& \multicolumn{9}{c}{$\begin{array}{c}\text { Elapsed time in seconds } \\
\text { (relative time to plain C) }\end{array}$} \\
& & Cilk & $\begin{array}{r}\text { Cilk w/o } \\
\text { SYNCHED }\end{array}$ & Tascell & $\begin{array}{r}\text { Tascell } \\
\text { w/ copying }\end{array}$ \\
\hline \hline Fib(40) & 0.926 & 7.15 & - & 2.30 & - \\
& $(1.00)$ & $(7.72)$ & & $(2.48)$ & \\
\hline Nqueens(15) & 10.3 & 29.8 & 37.1 & 15.8 & 25.4 \\
& $(1.00)$ & $(2.89)$ & $(3.60)$ & $(1.53)$ & $(2.47)$ \\
\hline Pentomino(12) & 1.68 & 2.37 & 2.74 & 2.19 & 2.80 \\
& $(1.00)$ & $(1.41)$ & $(1.63)$ & $(1.30)$ & $(1.67)$ \\
\hline LU(2000) & 8.66 & 8.54 & - & 8.69 & - \\
\hline Comp(30000) & $(1.00)$ & $(0.986)$ & & $(1.00)$ & \\
& $(1.00)$ & $(1.84$ & - & 5.42 & - \\
\hline Grav(200) & 3.35 & 7.01 & - & 4.55 & - \\
& $(1.00)$ & $(2.09)$ & & $(1.35)$ &
\end{tabular}

Table 2. Execution time (and relative time to sequential $\mathrm{C}$ programs) with one worker. In Nqueens and Pentomino of Cilk, we partially avoided needless allocation of workspaces by using SYNCHED. We used SYNCHED only for avoiding needless allocation; initialization of workspaces was always done by copying between workspaces. In "w/o SYNCHED", SYNCHED was not used; allocation was performed for every spawned logical thread. In "w/ copying" of Tascell, we performed artificial workspace allocation and copying between workspaces for each spawnable task.

zero overheads in both Cilk and Tascell because the potential task division is infrequent. lows:

The additional overheads in Cilk can be broken down as fol-

(a) cost of explicit frame management,

(b) cost of the THE protocol [5] for consistent access to the logical thread queue, and

(c) cost of copying between workspaces for each thread (for Pentomino and Nqueens).

The copying overhead can be estimated as the difference between Tascell programs with and without the artificial copying shown in Table 2. The effect of reusing allocated workspaces in Cilk with SYNCHED can be estimated as the difference shown in Table 2.

Figure 13 summarizes the results of performance measurements with multiple workers in a shared memory environment. In all benchmarks except LU, Tascell shows higher efficiency (see Figure 13's caption) than Cilk because of Tascell's lower serial overheads. For instance, we achieved a speedup of 1.86 times $(=0.692 / 0.372)$ as compared with Cilk in Nqueens(16) with 4 workers. Tascell's relative efficiency degradation in Fib with multiple workers is larger than Cilk's because Tascell's overheads for intranode communication are higher, although Tascell's absolute efficiency is considerably higher than Cilk's. The sudden efficiency drop in LU and Grav with 4 workers may be caused by memory bandwidth saturation.

The logarithmically scaled graphs in Figure 14 show the results of performance measurements on multiple computation nodes. ${ }^{3}$

When a single worker is running in each node (Figure 14 (a)), the programs except LU exhibit good speedups because their computation times are sufficiently long relative to the communication costs among computation nodes for the small numbers of spawned tasks; that is, the potential bottleneck around the Tascell server is insignificant in most applications. In contrast, we could not obtain the speedups in LU. To the best of our knowledge, it is diffi-

\footnotetext{
${ }^{3}$ We evaluated only Tascell because the standard implementation of Cilk only supports shared memory environments.
}

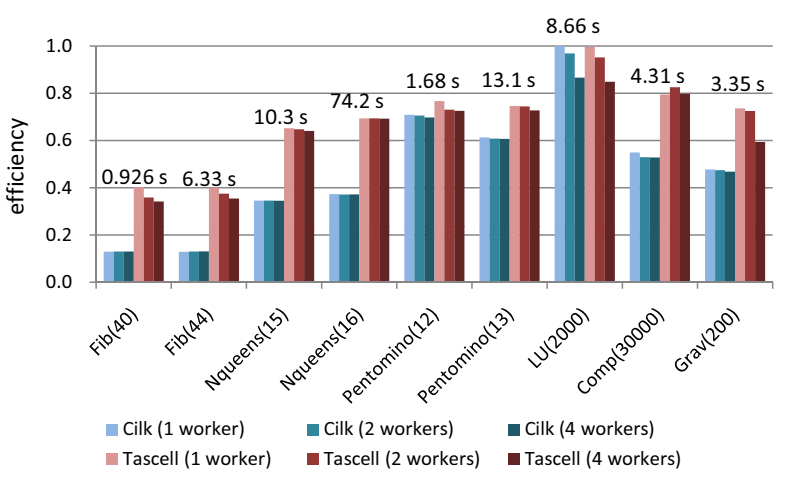

Figure 13. Efficiency with multiple workers in a shared memory environment within one node. Efficiency is defined as $S / n_{w}$ where $S$ is a speedup to a sequential C program and $n_{w}$ is the number of workers. (Efficiency $=1$ means an ideal speedup.) The number above each group of bars shows the execution time in $\mathrm{C}$.

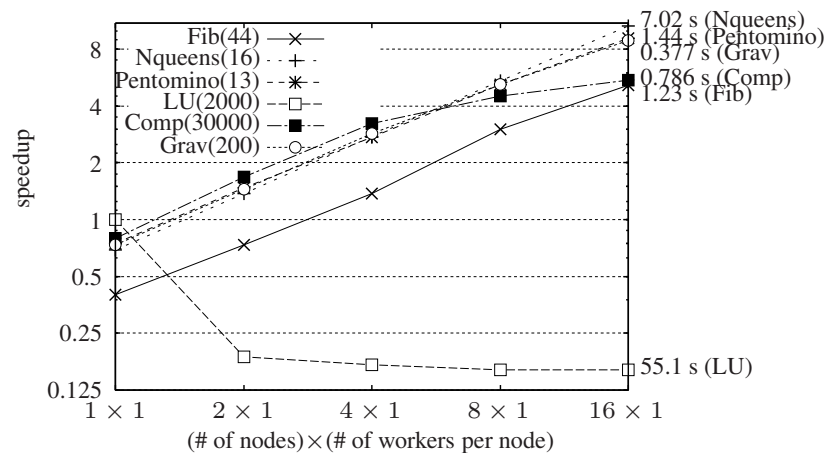

(a) one worker running in each node

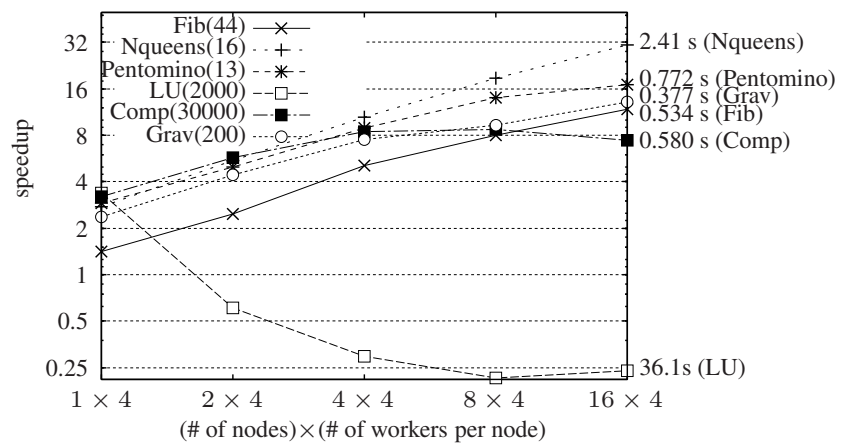

(b1) 4 workers running in each node

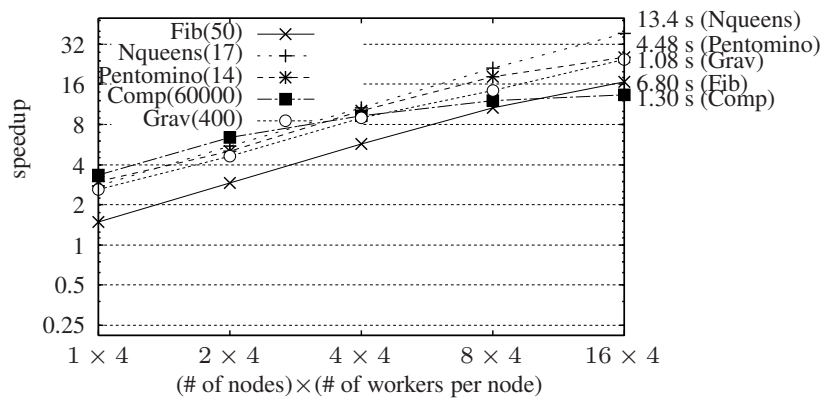

(b2) 4 workers running in each node (larger problems)

Figure 14. Speedups with multiple computation nodes (relative to sequential C). The numbers on the right side of each graph show execution time with 16 nodes. 
cult to obtain sufficient speedups in applications with large shared data such as LU with work-stealing-based dynamic load balancing without efficient support for (distributed) shared memory, as experienced also in [20], since dynamically stolen (transmitted) tasks/results must involve large submatrices.

In environments where multiple workers are running in each node (Figure 14 (b1), (b2)), the workers run with both internode and intranode communication. Figure 14 (b2) shows that we can get a good speedup also in such an environment as long as the size of a problem is sufficiently large relative to the number of workers (e.g., $>4$ s for each worker). The speedups in Comp are limited because transmission costs of $O(n)$ do not pay for small $n$ since the time complexity of Comp is $O\left(n^{2}\right)$.

We can improve the performance in distributed memory environments by improving the message handling of a Tascell server or employing some mechanism for sharing data among computation nodes.

\section{Conclusion and Future work}

We proposed a new scheme for dynamic load balancing on the basis of backtracking. Our scheme is useful especially for backtrack search algorithms where overheads are strongly reduced by delayed copying between workspaces, and we can write such algorithms elegantly. In addition, our scheme enables many applications to run more efficiently by allocating workspaces lazily and eliminating the cost of creating/managing logical threads. Furthermore, our taskobject-based parallel programming model enables programs to be easily written and executed for both shared and distributed (and also hybrid) memory environments.

We will try to improve the performance in distributed memory environments by implementing more sophisticated message handling among computation nodes or lazy and/or asynchronous data transmission, which will also alleviate potential bottlenecks around Tascell servers. We will also implement a mechanism to enable computation nodes to leave safely.

\section{Acknowledgments}

This work was partly supported by Grant-in-Aid for JSPS Fellows (192782) and MEXT Grant-in-Aid for Exploratory Research (17650008).

\section{References}

[1] Thomas M. Breuel. Lexical closures for C++. In Usenix Proceedings, C++ Conference, 1988.

[2] Philippe Charles, Christian Grothoff, Vijay Saraswat, Christopher Donawa, Allan Kielstra, Kemal Ebcioglu, Christoph von Praun, and Vivek Sarkar. X10: an object-oriented approach to non-uniform cluster computing. SIGPLAN Not., 40(10):519-538, 2005. ISSN 0362-1340.

[3] Marc Feeley. A message passing implementation of lazy task creation. In Proceedings of the International Workshop on Parallel Symbolic Computing: Languages, Systems, and Applications, number 748 in Lecture Notes in Computer Science, pages 94-107. Springer-Verlag, 1993.

[4] Marc Feeley. Lazy remote procedure call and its implementation in a parallel variant of C. In Proceedings of International Workshop on Parallel Symbolic Languages and Systems, number 1068 in Lecture Notes in Computer Science, pages 3-21. Springer-Verlag, 1995.

[5] Matteo Frigo, Charles E. Leiserson, and Keith H. Randall. The implementation of the Cilk-5 multithreaded language. ACM SIGPLAN Notices (PLDI '98), 33(5):212-223, 1998.

[6] Seth C. Goldstein, Klaus E. Schauser, and David E. Culler. Lazy Threads: Implementing a fast parallel call. Journal of Parallel and Distributed Computing, 3(1):5-20, August 1996.
[7] Robert H. Halstead, Jr. New ideas in parallel Lisp: Language design, implementation, and programming tools. In T. Ito and R. H. Halstead, editors, Parallel Lisp: Languages and Systems, volume 441 of Lecture Notes in Computer Science, pages 2-57, Sendai, Japan, June 5-8, 1990. Springer, Berlin.

[8] Tasuku Hiraishi, Masahiro Yasugi, and Taiichi Yuasa. A transformation-based implementation of lightweight nested functions. IPSJ Digital Courier, 2:262-279, 2006. (IPSJ Transaction on Programming, Vol. 47, No. SIG 6(PRO 29), pp. 50-67.).

[9] Intel Corporation. Intel Threading Building Block Tutorial, 2007. http://threadingbuildingblocks.org/.

[10] Richard Kelsey, William Clinger, and Jonathan Rees. Revised ${ }^{5}$ report on the algorithmic language Scheme. ACM SIGPLAN Notices, 33(9): 26-76, September 1998.

[11] B.C. Kuszmaul. Cilk provides the "best overall productivity" for high performance computing:(and won the HPC challenge award to prove it). Proceedings of the nineteenth annual ACM Symposium on Parallel Algorithms and Architectures, pages 299-300, 2007.

[12] Eric Mohr, David A. Kranz, and Robert H. Halstead, Jr. Lazy task creation: A technique for increasing the granularity of parallel programs. IEEE Transactions on Parallel and Distributed Systems, 2(3): 264-280, July 1991.

[13] Liang Peng, Weng Fai Wong, Ming Dong Feng, and Chung Kwong Yuen. SilkRoad: A multithreaded runtime system with software distributed shared memory for SMP clusters. In IEEE International Conferrence on Cluster Computing (Cluster2000), pages 243-249, November 2000.

[14] K. Randall. Cilk: Efficient multithreaded computing. Technical Report MIT/LCS/TR-749, 1998.

[15] R. M. Stallman. Using and porting GNU Compiler Collection. 1999.

[16] Volker Strumpen. Indolent closure creation. Technical Report MITLCS-TM-580, MIT, June 1998.

[17] Supercomputing Technologies Group. Cilk 5.4.6 Reference Manual. Massachusetts Institute of Technology, Laboratory for Computer Science, Cambridge, Massachusetts, USA.

[18] Kenjiro Taura, Kunio Tabata, and Akinori Yonezawa. StackThreads/MP: Integrating futures into calling standards. In Proceedings of ACM SIGPLAN Symposium on Principles \& Practice of Parallel Programming (PPoPP'99), pages 60-71, May 1999.

[19] Seiji Umatani, Masahiro Yasugi, Tsuneyasu Komiya, and Taiichi Yuasa. Pursuing laziness for efficient implementation of modern multithreaded languages. In Proceedings of the Fifth International Symposium on High Performance Computing, number 2858 in Lecture Notes in Computer Science, pages 174-188, October 2003.

[20] Rob V. van Nieuwpoort, Thilo Kielmann, and Henri E. Bal. Efficient load balancing for wide-area divide-and-conquer applications. In In Proceedings of the eighth ACM SIGPLAN symposium on Principles and Practices of Parallel Programming (PPoPP'01), pages 34-43, New York, NY, USA, 2001. ACM. ISBN 1-58113-346-4.

[21] Mark T. Vandevoorde and Eric S. Roberts. WorkCrews: An abstraction for controlling parallelism. International Journal of Parallel Programming, 17(4):347-366, 1988.

[22] David B. Wagner and Bradley G. Calder. Leapfrogging: A portable technique for implementing efficient futures. In Proceedings of Principles and Practice of Parallel Programming (PPoPP'93), pages 208217, 1993.

[23] Masahiro Yasugi, Tasuku Hiraishi, and Taiichi Yuasa. Lightweight lexical closures for legitimate execution stack access. In Proceedings of the Fifteenth International Conference on Compiler Construction (CC2006), number 3923 in Lecture Notes in Computer Science, pages 170-184. Springer-Verlag, 2006. 